\title{
Swiss DRGs: Patient Heterogeneity and Hospital Payments ${ }^{\mathrm{a}}$
}

\author{
Michel Mougeot $^{\mathrm{b}, \mathrm{c}}$ and Florence Naegelen $^{\mathrm{c}}$
}

JEL-Classification: I1, L3, D8

Keywords: hospital price regulation, outlier payment, piecewise mechanism

\section{Introduction}

As many European countries, Switzerland is currently implementing a prospective method of paying hospitals. Under this mechanism introduced in the U.S. in 1983, the payment rate per admission for a given diagnosis is selected before the treatment takes place. Each patient admitted to a hospital is classified according to its condition under one of Diagnosis Related Group (DRGs), each DRG representing a case type identifying patients with a similar diagnosis. When the payment is prospective, hospitals are paid a fixed amount per admission for a given DRG.

Formerly, hospitals were paid on a per diem and fee-for-service basis. These mechanisms were typical forms of retrospective reimbursement and gave the hospitals incentives to increase the length of stay and the units of service. Later, a global budget has been used to cap artificially the expense. Though global budgeting gives incentives to reduce the length of stay and the units of service, it was not linked to performance indicators and could involve rationing.

From an economic point of view, the prospective payment system is based on a fixed-price per patient treated. On the one hand, it gives the hospital socially optimal incentives to reduce costs and produce efficiently health care services

a This paper was presented at the annual meeting of the Swiss Society of Economics and Statistics at the University of Lausanne, on March 28, 2008. The analysis is based on a paper of M. Mougeot and F. Naegelen, forthcoming in the Journal of Risk and Insurance (December 2008).

b University of Franche-Comte, CRESE, UFR SJEPG, 45 D Avenue de l'Observatoire, 25030 Besançon Cedex, France.

c IEMS, University of Lausanne, Lausanne, Switzerland. E-mail address: michel.mougeot@ univ-fcomte.fr 
when cost reduction effort is non contractible. On the other hand, it can increase the number of cases when the fixed price per DRG is above marginal costs and decrease the level of health care quality services when patient demand does not reflect quality. ${ }^{1}$ Another drawback of this policy is a consequence of patient heterogeneity. When treatment costs depend on patient severity and when the within-DRG cost variance is high, hospitals may earn a substantial rent when treating a low cost patient. There is also a scope for dumping when facing a high cost patient (i.e., outlier patients).

In practice, in many countries, hospital payment system introduces retrospective payment for exceptionally costly patients. For very expensive cases, hospitals receive additional payments providing some compensation for unusually expensive admissions. These outlier payments are equal to a given percentage of the accounting cost above some threshold. Outlier cases are not paid at full cost but as outlier payments are tied to costs, they do not give incentives to reduce costs. This mixture of fully prospective payments for low costs patients and partially cost-based system for outlier patients makes the payment system lower powered than a pure fixed price system.

In practice, a regulator using this mechanism must design a fixed-price per DRG for the low cost patients, a cost sharing rule for the outliers and a threshold $\widehat{C}$ above which a patient is considered as an outlier. In many countries, the threshold is characterized by various methods based on the statistical distribution of the patients. For instance, in Belgium, Sweden, China and Denmark, it is based on the quartile range of the distribution of the length of stay whereas in Austria, Ireland or Australia, it is based on the standard deviation of the length of stay. In the APDRG Swiss System (version $6.0^{2}$ ), the threshold is such that outliers are $5.4 \%$ of the total. However, these statistical methods lack economic foundations.

How regulate hospitals using only these instruments? Two cases can be considered. When the supplier cannot observe the patients' severity, outlier payments serve as an insurance and protect hospitals against financial risk. This issue has been analyzed by Ellis and Mc Guire (1998) and by Keeler, Carter and TRUde (1998) in the absence of moral hazard. When moral hazard is present, Mougeot and NaEgelen (2008a) have shown that the best outlier payment policy is a cost reimbursement policy associated with an infinite penalty when the

1 See Chalkley and Malcomson (1998a). In contrast, when the number of patients wanting treatment is quality responsive, the first best level of quality may be achieved if the regulator designs an appropriate fixed price (see Ma (1994) and Chalkley and Malcomson (1998)).

2 See APDRG Suisse (2007). 
first best level of effort is not chosen by the hospital. When hospitals can identify high-cost patients, outlier payments protect patients against discrimination in admission. This issue has been considered by MA (1994) when rent extraction does not matter and when the cost reduction effort affects only the distribution of the less severe cases. Mougeot and Naegelen (2008) have shown how the optimal outlier payment policy must solve a trade-off between productive efficiency, rent extraction and dumping deterrence and how this optimal policy depends on the degree of altruism of the provider.

In this paper, we apply the method proposed by Mougeot and NAegelen (2008) to a specific setting in which the treatment cost is a convex function of the severity. We show that an outlier policy is not necessary when the hospital is sufficiently altruistic. On the contrary, when the hospital is less benevolent, the mixed policy must be preferred.

The paper is organized as follows. The basic trade-offs are presented is section 2. The model of Mougeot and Naegelen (2008) is analyzed in section 3 whereas the optimal payment policies are presented in section 4. Some conclusions are drawn in section 5 .

\section{The Basic Trade-Offs}

For simplicity, assume that the cost function for a given DRG can be written $\mathrm{C}=\beta-e$ where $\beta$ is the level of severity and $e$ the cost reduction effort. Exerting effort $e$ implies a positive disutility $\phi(e)$ Assume that neither $\beta$ nor $e$ is observable but that $C$ is ex post observable. Consequently, if the hospital regulation is based on a cost reimbursement policy $(p=C)$, a self-interested hospital maximizing its benefit $\pi=p-C-\phi(e)=-\phi(e)$ will chose a level of effort $e=0$ In this case, the cost reimbursement contract offers no incentive for cost reduction but is ideal for rent extraction because the hospital earns no rent. ${ }^{3}$ Moreover, there is no scope for dumping high cost patients because the hospital is reimbursed for its expense whatever the severity.

If the regulator designs a prospective fixed price $p$ for any level of severity the hospital will chose a level of effort $e^{*}$ maximizing $p-\beta+e-\phi(e)$ and such that the marginal benefit of effort (i.e. the marginal reduction of cost) is equal to its marginal disutility $\left(\phi^{\prime}(e)\right)$, which implies $1=\phi^{\prime}\left(e^{*}\right)$. Then the fixed-price contract induces the optimal amount of effort and productive efficiency is achieved. In

3 See Laffont and Tirole (1993). 
contrast, if $p$ is greater than $\beta-e^{*}$ and if the regulator can compensate the hospital for its effort, the supplier can earn a rent when treating a low cost patient. Moreover, some high cost patients may be dumped if the fixed price is lower than their cost.

If health expenditure is financed by distortionary taxation, the rent is socially costly. Then, under asymmetric information, choosing an optimal contract implies solving a trade-off between rent extraction and productive efficiency. In this case, the optimal mechanism is an incentive contract tailored to the hospital's information on patients' severity. ${ }^{4}$

As regulators are reluctant to use incentive cost sharing contracts (see CHALKLeY and Malcomson (2002)), we must consider the trade-off between rent extraction, productive efficiency and dumping deterrence when the policy tools are only the fixed price and the cost sharing rule for the outliers.

Figure 1: Fixed-Price Policy

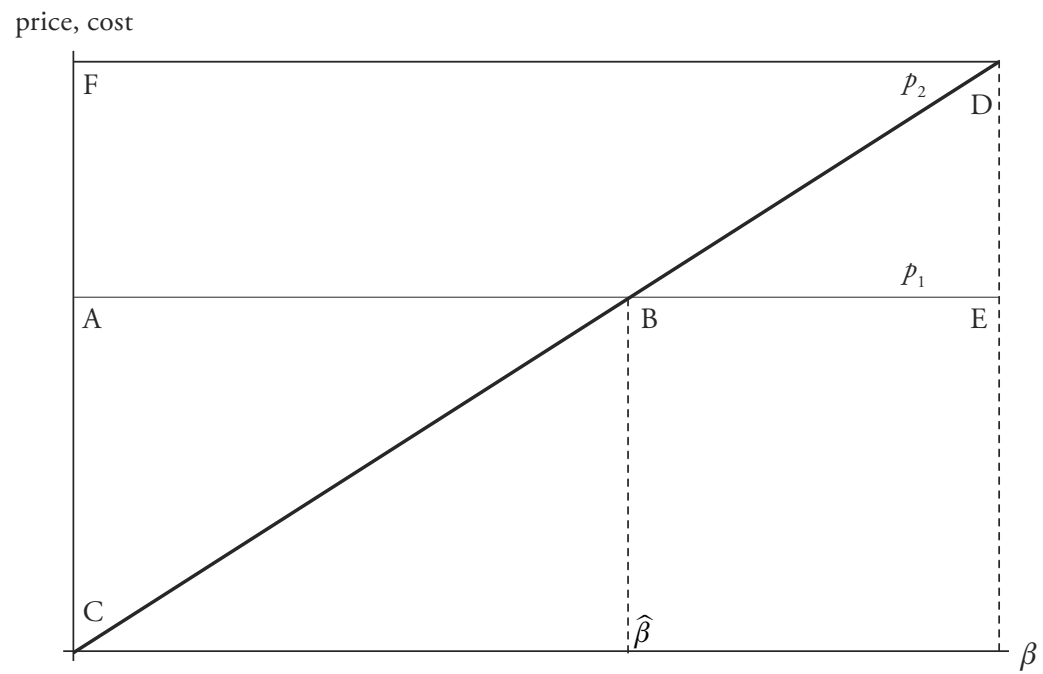

Consider firstly the case of the fixed-price. ${ }^{5}$ Assume $\beta \in[\beta, \bar{\beta}]$. If $p=p_{1}$, a selfinterested hospital exerts the first best level of effort $e^{*}$, earns a rent $\mathrm{ABC}$ on all patients with severity lower than $\widehat{\beta}$ and can refuse treatment to all patients with

4 It is an application of the results of Laffont and Tirole (1993).

5 See Figure 1. 
severity higher than $\widehat{\beta}$. An altruistic hospital can treat all the patients if the rent $\mathrm{ABC}$ on low cost patients offsets the loss BDE on high cost patients. If the price is equal to $p_{2}$, all patients are treated by a self-interested hospital but the socially costly rent is equal to the area FDC.

Assume now that the regulator designs an outlier payment policy with a threshold $\widehat{\beta}$ and a cost sharing rule $0 \leq a<1$ for the outliers. For a patient with severity $\beta>\widehat{\beta}$, the hospital will exert a cost reduction effort $e^{0}<e^{*}$. Then the cost $C\left(e^{o}\right)$ will be higher than $C\left(e^{*}\right)$. As the hospital receives an additional payment for treating patients with a high severity, the regulator can reduce the fixed price that should be equal to the cost of the patient with the highest severity under a fixed-price policy. Then it reduces the rent earned on patients with a low severity. However, introducing a cost sharing rule implies an efficiency loss because the effort exerted on patients with a high severity is lower than $e^{*}$. Consequently the optimal policy must trade off this loss with the rent reduction. This trade-off depends on the hospital's degree of concern for the patient benefit. If it is high, the hospital can agree to losses on high cost patients being offset by gains on low cost patients, provided budget is balanced. If it is low, the hospital does not refuse treatment to high cost patients only if it incurs no loss on these patients.

\section{The Model}

Let us consider a hospital treating patients with a given diagnosis. Each patient is characterized by the severity of her illness $\beta$. $\beta$ is observed by the hospital but the regulator does not know the value of the severity of a given patient. It only knows that $\beta$ is distributed over $[\underline{\beta}, \bar{\beta}]$ according to a cumulative distribution function $F($.) with a continuously differentiable density function $f()>$.0 , such that $F(.) / f($.$) is non decreasing in \beta .^{6}$

Assume that the cost to the hospital of treating a type $\beta$ patient is $C=c+\beta^{3}-e$ where $e$ is the cost reduction effort that the hospital manager can exert and $c$ is a common knowledge cost common to all patients. ${ }^{7}$ When the hospital can exert effort level $e$, it incurs a disutility $\phi(e)=e^{3} / 3$. The regulator does not monitor $e$ but observes the ex-post treatment cost $C$. Then the optimal policy is subject to moral hazard and adverse selection.

6 This assumption ensures that second order conditions are satisfied in our model.

7 Our insights carry over to more general settings. 
In the following, ${ }^{8}$ we look for the optimal contract that a payer could design when the set of policy tools it can use is restricted to the APDRG Swiss System? instruments, i.e.,

- for patients with cost lower than or equal to a threshold $\widehat{C}$, a fixed price $p$ is paid to the hospital,

- for outlier patients, i.e. patients with cost higher than a threshold $\widehat{C}$, the hospital receives a payment $t=p+a(C-\widehat{C})$ where $C$ is the realized cost and $a$ is a cost-sharing parameter, with $0 \leq a<1$.

We assume that the hospital is partially benevolent and trades off its benefit and the benefit for the patients. We denote $\alpha$ the degree to which the hospital takes the patient's interest $V(\beta)$ into account. In the case of a profit maximizing hospital, this ethical parameter is equal to 0 .

Under this outlier payment policy, hospital utility can be written, when a patient of type $\beta$ is treated and when the hospital exerts an effort level $e$ :

$$
\begin{gathered}
U_{p}(p, \beta, e)=p-c-\beta^{3}+e-\phi(e)+\alpha V(\beta) \text { if } c+\beta^{3}-e \leq \widehat{C} \\
U_{t}(p, \beta, e, \widehat{C}, a)=p+(a-1)\left(c+\beta^{3}-e\right)-a \widehat{C}-\phi(e)+\alpha V(\beta) \\
\text { if } c+\beta^{3}-e>\widehat{C}
\end{gathered}
$$

The form of $V(\beta)$ is an open question. $V(\beta)$ may be increasing or decreasing. However, we assume that $U^{\prime}(\beta)<0$ for all $\beta$, which implies that monetary plus non monetary cost rises with $\beta$ faster than benefit $V(\beta) .{ }^{10}$ Normalizing the hospital's outside opportunity level of utility to 0 , the hospital's individual rationality constraint is $U(\beta) \geq 0$.

When $c+\beta^{3}-e \leq \widehat{C}$, the hospital faces the fixed-price contract and chooses a first best level of cost reduction effort $e^{*}=1$ whereas when $c+\beta^{3}-e>\widehat{C}$, the hospital chooses a level of cost reduction effort $e^{o}(a)=\sqrt{1-a}$. In MOUgEot and NAEgelen (2008), we show that the hospital treating a patient with severity $\beta$ can influence the payment rule only by changing its effort and that there exists

8 See Mougeot and Naegelen (2008) for a detailed analysis.

9 The APDRG Swiss System includes also a specific rule for the "low" outliers. We do not consider this rule in this paper. Moreover, in practice, the costs are evaluated according to the length of stay

10 If $V(\beta)$ is decreasing with $\beta$ (as in De Fraja (2000)), it is always true. If the payer takes more care of high cost patients in its objective, it is satisfied for some functions $V(\beta)$. 
a unique value $\widehat{\beta}$ such that the hospital is indifferent between the fixed price and the outlier payment with

$$
\widehat{\beta}=\left(\widehat{C}-c+e^{o}+\frac{e^{*}-\phi\left(e^{*}\right)-e^{o}+\phi\left(e^{o}\right)}{a}\right)^{1 / 3}
$$

We assume that the payer attaches benefit $V(\beta)$ to having a patient with severity $\beta$ treated and maximizes an expected utilitarian social welfare function $E W$ equal to the expected value of the sum of the net benefit of the treatment and the hospital's utility $U(e, \beta)$. As health care expenditure is financed by distortionary taxes, ${ }^{11}$ transfers from the regulator to the hospital have to be multiplied by $1+\lambda$ where $\lambda>0$ is the shadow cost of public funds. When the payer designs the Medicare payment scheme, expected welfare can be written

$$
\begin{aligned}
E W= & \beta E V(\beta)-\lambda p-c-E\left(\beta^{3}\right)+e^{*}-\phi\left(e^{*}\right)+\lambda a \int_{\widehat{\beta}}^{\bar{\beta}} 3 \beta^{2} F(\beta) d \beta \\
& +(1-F(\widehat{\beta}))(1+\lambda)\left(e^{o}(a)-\phi\left(e^{o}(a)\right)-e^{*}+\phi\left(e^{*}\right)\right)+\lambda a\left(\widehat{\beta}^{3}-\bar{\beta}^{3}\right)
\end{aligned}
$$

When designing the outlier payment policy, the payer must choose a threshold $\widehat{\beta} \leq \bar{\beta}$, a price $p$ and a cost sharing parameter $a, 0 \leq a \leq 1$ maximizing $E W$ in (4) subject to three sets of constraints:

- the participation (or no dumping) constraints with respect to the lower cost patients. For all patients with severity $\beta \leq \widehat{\beta}$, hospital utility must be non negative:

$$
\begin{gathered}
U_{p}(p, \beta)=p-c-\beta^{3}+e^{*}-\phi\left(e^{*}\right)+\alpha V(\beta) \geq 0 \\
\forall \beta \leq \widehat{\beta}
\end{gathered}
$$

- the participation constraints with respect to the outlier patients. For all outlier patients to be treated, hospital utility must be non negative. Then, from (2) and (5):

$$
\begin{gathered}
U_{t}(p, \beta, \widehat{\beta}, a)=p-c+e^{*}-\phi\left(e^{*}\right)+(a-1) \beta^{3}-a \widehat{\beta}^{3}+\alpha V(\beta) \geq 0 \\
\forall \beta \geq \widehat{\beta}
\end{gathered}
$$

11 This is partially true in Switzerland. 
- the expected budget constraint including hospital's manager compensation

$$
\begin{aligned}
B(p, \widehat{\beta}, a)= & p-c-E\left(\beta^{3}\right)+e^{*}-\phi\left(e^{*}\right) \\
& -a \int_{\widehat{\beta}}^{\bar{\beta}} 3 \beta^{2} F(\beta) d \beta+a\left(\bar{\beta}^{3}-\widehat{\beta}^{3}\right) \geq 0
\end{aligned}
$$

As $U(\beta)$ is decreasing in $\beta$, the sets of constraints (5) and (6) can be replaced with

$$
U_{t}(p, \bar{\beta}, e, \widehat{C}, a) \geq 0
$$

\section{Optimal Mechanisms}

In Mougeot and Naegelen (2008), we show that three cases must be distinguished according to the degree of benevolence of the supplier.

\subsection{Strongly Benevolent Hospital}

When the budget constraint (7) is binding and the no-dumping constraint (8) is slack, the optimal mechanism is a fixed price mechanism with a price equal to the mean value of the total cost: $p_{1}=c+E \beta^{3}-e^{*}+\phi\left(e^{*}\right)$. As the participation constraint is not binding, we must have $\alpha V(\bar{\beta})>\bar{\beta}-E \beta^{3}$. This is the case of a high degree of concern for the patient benefit. Altruism reduces the hospital's willingness to dump high cost patients even if it is not reimbursed for its cost, provided budget is balanced. As the fixed price is equal to the mean value of the total cost $C\left(e^{*}\right)+\phi\left(e^{*}\right)$ under optimal effort, high cost patients are subsidized by low cost patients. In this case, there is no trade-off between rent extraction, productive efficiency and dumping deterrence.

To illustrate this result, let us assume that $c=1, \lambda=1 / 4$ and that the severity parameter $\beta$ is distributed on $[0,1]$ according to a Beta distribution with parameter $m=5$ and $n=3$. The density function is given by $f(\beta)=105 \beta^{4}(1-b)^{2}$ and the cumulative distribution function is $F(\beta)=21 \beta^{5}-35 \beta^{6}+15 b^{7}$. The strongly benevolent case occurs if $\alpha V(\bar{\beta})>0.708$. The fixed-price $p_{1}$ is equal to 0.625 . This mechanism and total cost are represented in Figure 2. 
Figure 2: Strongly Benevolent Hospital

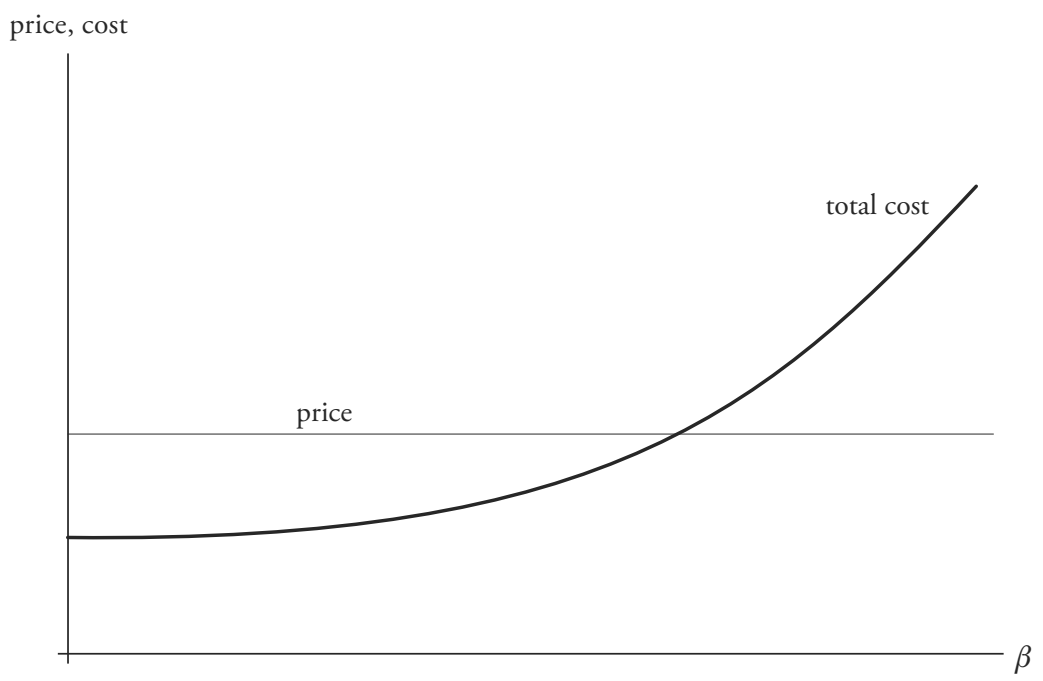

\subsection{Weakly Benevolent Hospital}

When the participation constraint is binding and the budget constraint is lacking, the optimal mechanism is characterized by a threshold $\widehat{\beta}_{2}$, a cost-sharing rule $a_{2}$ for the outlier patients and by a fixed price

$$
p\left(\widehat{\beta}_{2}\right)=c+\bar{\beta}^{3}-e^{*}+\phi\left(e^{*}\right)-\alpha V(\bar{\beta})+a_{2}\left(\widehat{\beta}_{2}^{3}-\bar{\beta}^{3}\right)
$$

for the other patients, where $a_{2}$ and $\widehat{\beta}_{2}$ are solutions of

$$
\begin{gathered}
-3 \lambda a \widehat{\beta}^{2} F(\widehat{\beta})+f(\widehat{\beta})(1+\lambda)\left(e^{*}-\phi\left(e^{*}\right)-e^{o}(a)+\phi\left(e^{o}(a)\right)\right)=0 \\
a \frac{d\left(e^{o}(a)\right)}{d a}(1+\lambda)(1-F(\widehat{\beta}))+\lambda \int_{\widehat{\beta}}^{\bar{\beta}} 3 \beta^{2} F(\beta) d \beta=0
\end{gathered}
$$

This happens when

$$
\alpha V(\bar{\beta})<\bar{\beta}-E \beta^{3}-a_{2} \int_{\widehat{\beta}_{2}}^{\bar{\beta}} 3 \beta^{2} F(\beta) d \beta .
$$


This is the case of a low degree of concern for patients' benefit. This mechanism implies a lower rent on the patients with a low severity but a higher cost when treating the high costs patients. This is the result of a trade-off between rent extraction, productive efficiency and dumping deterrence.

Under our previous assumptions, $p_{2}=0.816, a=0.852, \widehat{\beta}=0.856, \widehat{C}=0.89$ for $\alpha V(\bar{\beta})<0.397$. If we assume that $\alpha V(\bar{\beta})=0.2$, the optimal mechanism is a fixed-price $p_{2}=0.816$ for patients with cost lower than 0.89 and a cost sharing rule $t_{2}=0.816+0.852(C-0.89)=0.816+0.852\left(\beta^{3}-0.275\right)$ for the outliers whose cost is greater than $\widehat{C}$. This mechanism and total cost are represented in Figure 3.

Figure 3: Weakly Benevolent Hospital

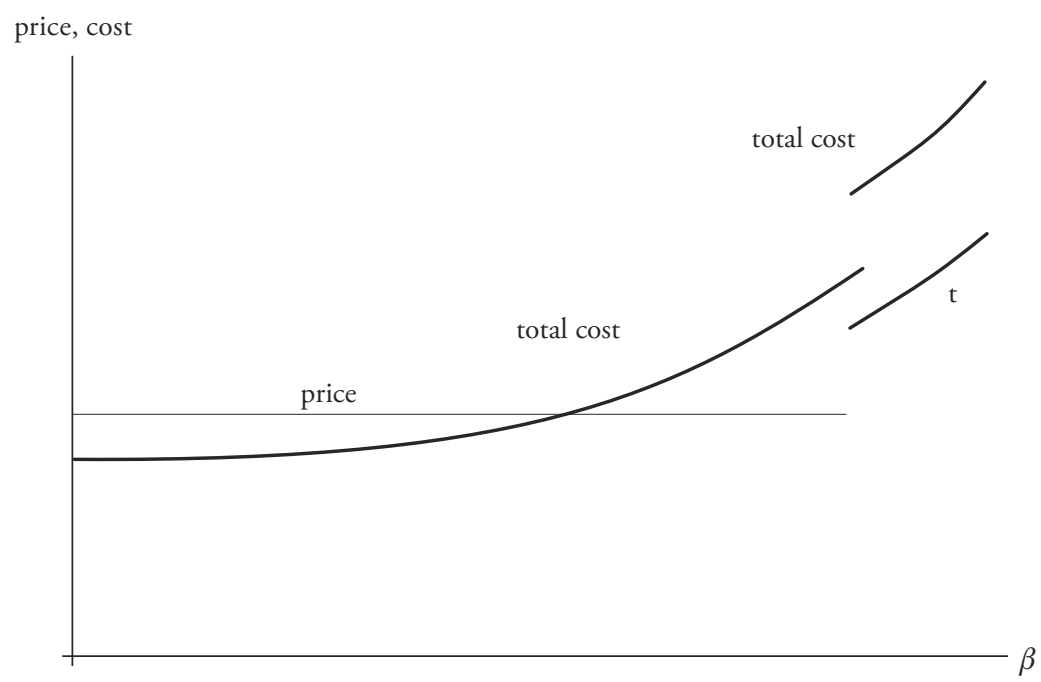




\subsection{Intermediate Degree of Benevolence}

When both constraints are binding, the optimal mechanism is characterized by a threshold $\widehat{\beta}_{3}$, a cost-sharing rule $a_{3}$ for the outlier patients and by a fixed price

$$
p\left(\widehat{\beta}_{3}\right)=c+\bar{\beta}^{3}-e^{*}+\phi\left(e^{*}\right)-\alpha V(\bar{\beta})+a_{3}\left(\widehat{\beta}_{3}^{3}-\bar{\beta}^{3}\right)
$$

for the other patients, where $a_{3}$ and $\widehat{\beta}_{3}$ are solutions of

$$
\begin{gathered}
a_{3}^{2} \frac{\partial e^{o}}{\partial a}\left(1-F\left(\widehat{\beta}_{3}\right)\right) \\
+\frac{\left(e^{*}-\phi\left(e^{*}\right)-e^{o}(a)+\phi\left(e^{o}(a)\right)\right) f\left(\widehat{\beta}_{3}\right)}{F\left(\widehat{\beta}_{3}\right)} \int_{\widehat{\beta}_{3}}^{\bar{\beta}} 3 \beta^{2} F(\beta) d \beta=0 \\
a_{3} \int_{\widehat{\beta}_{3}}^{\bar{\beta}} 3 \beta^{2} F(\beta) d \beta-\bar{\beta}+E \beta^{3}+\alpha V(\bar{\beta})=0
\end{gathered}
$$

This happens when

$$
\bar{\beta}-E \beta^{3}-a_{2} \int_{\widehat{\beta}_{2}}^{\bar{\beta}} 3 \beta^{2} F(\beta) d \beta<\alpha V(\bar{\beta})<\bar{\beta}-E \beta^{3} .
$$

As the expected budget is balanced, the payer has only to solve a trade-off between efficiency and dumping deterrence. Consequently, $\widehat{\beta}_{3}$ and $a_{3}$ do not depend on the social cost of public funds.

Under our assumptions, the intermediate case occurs when $0.397<\alpha V(\bar{\beta})$ $<0.708$. Let us assume that $\alpha V(\bar{\beta})=0.5$. Then $p_{3}=0.624, a=0.835, \widehat{\beta}$ $=0.908, \widehat{C}=1$. The optimal policy is a fixed price equal to 0.624 for patients with $C<1$ and the cost-sharing rule

$$
t_{3}=0.624+0.835(C-1)=0.624+0.835\left(\beta^{3}-0.410\right)
$$

for the outliers whose cost is greater than $\widehat{C}=1$. This mechanism and total costs are represented in Figure 4. 
Figure 4: Intermediate Degree of Concern for the Patient's Benefit

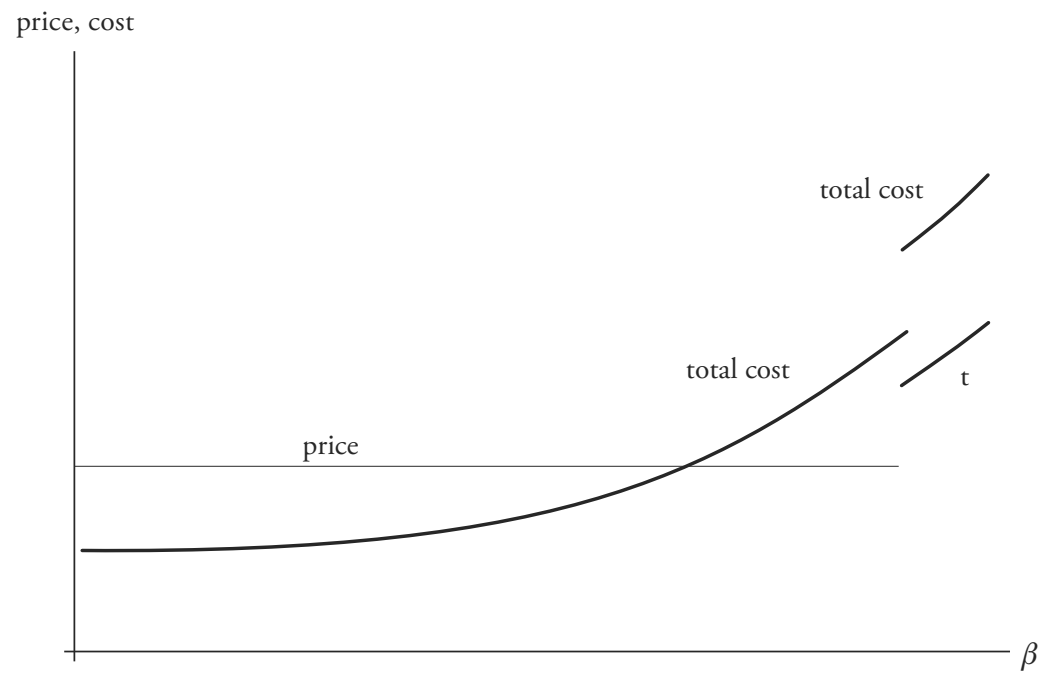

\section{Conclusion}

Our analysis shows how the optimal hospital price regulation depends on the degree $\alpha$ to which the hospital takes the patient's interest into account. Under a fixed-price mechanism, the hospital can dump costly patients and earns a rent on the low cost patients. When the degree of altruism is high, the outlier issue vanishes because the supplier agrees to cross subsidize between high costs and low costs. When the degree of altruism is low, the regulator must leave a monetary rent to the hospital. If we assume that a for-profit hospital has a low degree of benevolence, an outlier payment policy is necessary.

As in many papers on hospital pricing policy (Chalkley and Malcomson (1998), Ellis and Mc Guire (1986), among others), the principal must design contracts depending on the degree of altruism, which implies that this degree is common knowledge. When agents are altruistic or public-service motivated, this parameter becomes a policy instrument. JACK (2005) assumes that it is private information and derives conditions for the optimal revealing mechanism. A twodimensional private information model could consider simultaneously the non observability of the provider's degree of altruism and of the patient severity. 


\section{References}

APDRG Suisse (2007), Cost-Weights Version 6.0 ISE, Ecublens, May.

Chalkley, M., and J. M. Malcomson (1998), "Contracting for Health Services with Unmonitored Quality”, Economic Journal, 108, pp. 1093-1110.

Chalkley, M., and J. M. Malcomson (1998a), "Contracting for Health Services when Patient Demand Does not Reflect Quality”, Journal of Health Economics, 17(1), pp. 1-19.

Chalkley, M., and J. M. Malcomson (2002), "Cost Sharing in Health Service Provision : An Empirical Assessment of Cost Savings", Journal of Public Economics, 84, pp. 219-249.

Ellis, R. P., and Th. McGuire (1986), "Provider Behavior under Prospective Reimbursement: Cost Sharing and Supply", Journal of Health Economics, 5, pp. 129-152.

Ellis, R. P, and Th. McGuire (1988), "Insurance Principles and the Design of Prospective Payment System", Journal of Health Economics, 7(3), pp. 215-237.

JACK, W. (2005), "Purchasing Health Care Services from Providers with Unknown Altruism", Journal of Health Economics , 24, pp. 73-93.

Keeler, E. B., G. Carter and S. Trude (1988), "Insurance Aspect of DRG Outlier Payments", Journal of Health Economics, 7(3), pp. 193-214.

Laffont, J. J., and J. Tirole (1993), A Theory of Incentives in Regulation and Procurement, Cambridge, MIT Press,

Ma C. T. A. (1994), "Health Care Payment Systems: Cost and Quality Incentives", Journal of Economics and Management Strategy, 3(1), pp. 93-112

Mougeot, M., and F. Naegelen (2008), "Adverse Selection, Moral Hazard and Outlier Payment Policy", The Journal of Risk and Insurance, forthcoming.

Mougeot, M., and F. Naegelen (2008a), "Supply-Side Risk Adjustment and Outlier Payment Policy", Journal of Health Economics, forthcoming

\section{SUMMARY}

In this paper, we analyze the prospective method of paying hospitals when the within-DRG variance is high. To avoid patients dumping, an outlier payment system is implemented. In the APDRG Swiss System, it consists in a mixture of fully prospective payments for low costs patients and partially cost-based system for high cost patients. We show how the optimal policy depends on the degree to which hospitals take patients' interest into account. A fixed-price policy is 
optimal when the hospital is sufficiently benevolent. When the hospital is weakly benevolent, a mixed policy solving a trade-off between rent extraction, efficiency and dumping deterrence must be preferred. Following Mougeot and Naegelen (2008), we show how the optimal combination of fixed price and partially costbased payment depends on the degree of benevolence of the hospital, the social cost of public funds and the distribution of patients severity. 